\title{
EXISTENCE D'APPLICATIONS HARMONIQUES TORDUES À VALEURS DANS LES VARIÉTÉS À COURBURE NÉGATIVE
}

\author{
FRANCOIS LABOURIE
}

(Communicated by Jonathan M. Rosenberg)

\begin{abstract}
We generalize a theorem of Corlette on existence of twisted harmonic maps into a manifold of non-positive curvature.
\end{abstract}

Ce papier fait suite à une suite d'articles $[D, C]$, étudiant les applications harmoniques tordues. Le problème est le suivant:

On se donne une variété $N$, dont le revêtement universel est $\bar{N}$, une variété $M$ simplement connexe à courbure négative ou nulle, et une représentation $\rho$ de $\pi_{1}(N)$ dans le groupe des isométries de $M$. Une application tordue par $\rho$ est alors une application de $\bar{N}$ dans $M \rho$-équivariante, ce qui peut s'interpréter comme une section du $M$-fibré plat de base $N$ et associé naturellement à $\rho$. Il s'agit maintenant d'obtenir une condition sur $\operatorname{Im}(\rho)$ permettant d'assurer l'existence d'une application harmonique tordue par $\rho$. Par exemple, $\operatorname{Im}(\rho)$ cocompact suffit selon les résultats célèbres de Eells et Sampson [ES].

Le résultat principal de Corlette répond à cette question dans le cas où $M$ est un espace riemannien symétrique de type noncompact:

Théorème 0.1 (Corlette). Il existe une application harmonique tordue par $\rho$ à valeur dans $G / K$ si et seulement si $\operatorname{Im}(\rho)$ est un sous-groupe réductif de $G$.

Corlette utilise ce résultat comme un point de départ de sa théorie de la superrigidité. Dans ce même article, il s'interroge sur une possible généralisation de ce résultat et une démonstration plus liée à la géométrie des variétés à courbure négative. Nous nous proposons ici d'en donner une extension, modulo une condition sur la cible $M$, à laquelle on demande d'être sans demies-bandes plates (1.1). Cette condition, quoique restrictive, est satisfaite par exemple dans le cas des variétés analytiques reélles ou des variétés à courbure strictement négative. Moyennant une définition géométrique de la condition de réductivité (1.3), nous obtenons la généralisation suivante du théorème de Corlette:

Théorème 0.2. Si $M$ est sans demies-bandes plates, il existe une application harmonique tordue par $\rho$, si et seulement si $\operatorname{Im}(\rho)$ est un sous-groupe réductif des isométries de $M$.

Received by the editors October 31, 1989 and, in revised form, April 18, 1990.

1980 Mathematics Subject Classification (1985 Revision). Primary 58E20; Secondary 58D25, $53 \mathrm{C} 20,53 \mathrm{C} 35$. 
Remarquons, que la condition de réductivité reste suffisante si l'on supprime l'hypothèse "sans demies-bandes plates" (3.4).

La démonstration de Corlette repose sur l'étude d'une équation d'évolution sur la connexion induite sur un fibré associé. La nôtre réutilise les résultats de [ES], et la géométrie des variétés à courbure négative ou nulle (sphère à l'infini, horosphères et flots horosphériques), telle qu'elle est étudiée dans [E0] et [BGS] par exemple.

\section{DÉFINITIONS}

Les variétés à courbure négative ou nulle qui nous intéressent sont celles qui satisfont au critère suivant:

1.1. Bandes plates. Une variété simplement connexe à courbure négative ou nulle est dite sans demies-bandes plates, si tout champ de Jacobi, défini le long d'une géodésique, dont la norme est constante pour les temps supérieurs à un temps donné, a une norme constante pour tout temps.

1.2. Remarques. (i) Cette propriété est la version infinitésimale de la propriété suivante: toute demie-bande plate peut être étendue en une bande plate entière.

(ii) Les variétés à courbure strictement négative, les variétés analytiques réelles et en particulier par les espaces symétriques sont sans demies-bandes plates.

Nous allons finir de donner les définitions nécessaires à l'énoncé de notre résultat en définissant un

1.3. Groupe réductif. Un sous-groupe $G$ du groupe des isométries d'une variété simplement connexe $M$ à courbure négative ou nulle est réductif s'il existe un convexe fermé $C$ de $M$, fixé globalement par $G$, tel que

(1) le convexe $C$ est isométrique à $C_{1} \times E$, où $E$ est euclidien,

(2) $G$ se décompose en $G=G_{1} \times G_{2}, G_{1}$ agissant isométriquement sur $C_{1}$ et $G_{2}$ sur $E$.

(3) $G_{1}$ ne fixe pas de point à l'infini de $C_{1}$.

1.4. Remarques. (i) Dans le cas où $M$ est analytique réelle, on peut remplacer le mot convexe, par l'expression totalement géodésique et complet (ce qui inclut les points...);

(ii) Dans le cas où $M$ est à courbure strictement négative, un sous-groupe est réductif exactement dans l'un des cas suivants:

(1) il ne fixe pas de point à l'infini de $M$,

(2) il fixe globalement une géodésique.

Autrement dit, dans ce cas, un groupe est non réductif si et seulement si il fixe un unique point à l'infini. 


\section{DANS UN SENS}

Dans cette section, nous allons démontrer, en utilisant les notations de l'introduction, que s'il existe une application harmonique $f$, tordue $\operatorname{par} \rho$, de $\bar{N}$ dans $M$, alors $G=\operatorname{Im}(\rho)$ est un sous-groupe réductif des isométries de $M$.

Soit donc $p$ le plus grand entier tel qu'il existe un convexe fermé $C$ de $M$, fixé globalement par $G$, vérifiant

(1) $C$ est isométrique à $C_{1} \times E$ où $E$ est euclidien de dimension $p$ (On identifiera $C_{1}$ à un convexe de $M$ );

(2) $G$ se décompose en $G=G_{1} \times G_{2}, G_{1}$ agissant isométriquement sur $C_{1}$ et $G_{2}$ sur $E$.

Nous voulons montrer que $G_{1}$ ne fixe pas de point à l'infini de $C_{1}$.

Démontrons tout d'abord le

Lemme. Soit $\pi$ la projection sur le convexe $C_{1}$, et $\rho_{1}$ la représentation de $\pi_{1}(N)$ induite par $\rho$ et la projection de $G$ sur $G_{1}$, alors $\pi \circ f$ est une application harmonique de $\bar{N}$ dans $C_{1}$, tordue par $\rho_{1}$.

Preuve. Nous allons tout d'abord montrer que si $\pi_{0}$ est la projection convexe sur $C$, alors $g_{0}=\pi_{0} \circ f$ est harmonique. Remarquons premièrement que, la fonction distance à un convexe étant convexe en courbure négative [BGS], sa restriction à $f(\bar{N})$ est contante. Autrement dit $f$ est à valeur dans l'hypersurface $S_{t_{0}}$ bord du convexe

$$
C_{t_{0}}=\left\{m \in M \mid d(m, C) \leq t_{0}\right\} .
$$

Notons $g_{t}=\pi_{t} \circ f$, ou $\pi_{t}$ est la projection sur $C_{t}$ et remarquons que ces applications sont toutes tordues par $\rho$.

Soit $u$ un vecteur tangent à $\bar{N}$ en $x$ et $v_{t}=D g_{t}(u)$, par construction $v_{t}$ est un champ de Jacobi le long de la géodésique $g_{t}(x)$.

$M$ étant à courbure négative et la projection sur un convexe étant contractante, nous en déduisons que la norme de $v_{t}$ est convexe et croissante. Par ailleurs, $f$ étant harmonique, nous en déduisons que cette fonction à une dérivée nulle en $t=t_{0}$ et donc qu'elle est constante.

Les vecteurs $D g_{t}(u)$ sont donc des champ de Jacobi parallèles, ce qui entraîne que le champ, défini le long de la géodésique $g_{t}(x)$, des vecteurs de tension des $g_{t}$ est parallèle et donc constamment nul, puisque $f$ est harmonique.

Dès lors $g_{t}$ est harmonique pour tout $t$.

Maintenant, il est clair que la projection $g=\pi \circ f$ est harmonique et tordue $\operatorname{par} \rho_{1}$.

Terminons notre preuve en raisonnant par l'absurde. Supposons que $G_{1}$ fixe un point à l'infini sur $C_{1}$ et soit $\phi_{t}$ le flot géodésique associé à ce point à l'infini. Par construction, il est invariant par $G_{1}$, et donc les applications $g_{t}=\phi_{t} \circ g$ sont tordues par $\rho_{1}$. 
Notons à nouveau $v_{t}=D g_{t}(u)$, où $u$ est un vecteur tangent à $\bar{N}$. Comme dans le lemme précedent, $v_{t}$ est un champ de Jacobi dont la norme est une fontion convexe et décroissante. Or à nouveau comme $g$ est harmonique nous en déduisons que la dérivée de cette norme est nulle en $t=0$.

Ceci entraîne que cette norme est constante pour tout temps positif, et donc que, $M$ étant sans demies-bandes plates, cette norme est contante pour tout temps.

Dès lors si $x$ et $y$ deux points de $\bar{N}$, les géodésiques $g_{t}(x)$ et $g_{t}(y)$ sont parallèles. Nous en déduisons que l'ensemble $F$ des géodesiques parallèles à $g_{t}(x)$ est stable par $G_{1}$. Or d'après [G], cet ensemble est isométrique à $C_{2} \times \mathbf{R}$, où $C_{2}$ est un convexe de $M$. Par ailleurs $G_{1}$ commute avec les translations sur le facteur euclidien et tout ceci entraine donc que la décomposition initiale associée à la réduction de $G$ peut se poursuivre et donc que $p$ n'est pas le plus grand entier possible.

Ceci nous fournit notre contradiction.

\section{DANS L'AUTRE SENS}

Nous allons maintenant démontrer que si $\operatorname{Im}(\rho)$ est réductif, il existe une application harmonique tordue par $\rho$ de $\bar{N}$ dans $M$.

Ce résultat découlera bien évidemment des deux lemmes suivants, qui tout deux partent de la même idée que celle de la démonstration de Donaldson dans [D].

Lemme A. Soit $C$ un convexe fermé d'une variété à courbure négative, si $\operatorname{Im}(\rho)$ preserve $C$ et ne fixe pas de points à l'infini de $C$, alors il existe une application harmonique tordue par $\rho$.

Lemme B. Si $M$ est plate, alors il existe une application harmonique tordue par $\rho$.

3.1. Remarque. Le point de départ de ces deux lemmes, est l'utilisation, comme dans $[E S]$ et $[D]$, de l'équation d'évolution:

$$
\partial f_{t} / \partial t=-d_{\nabla}^{*}\left(D f_{t}\right),
$$

où $d_{\nabla}^{*}$ désigne l'adjoint de la dérivation extérieure des 1-formes de $\bar{N}$ à valeurs dans $f^{*}(T M)$, associée aux connexions de Levi-Cività de $M$ et $N$.

Partant d'une application $f_{0}$ de $\bar{N}$ dans $M$ tordue par $\rho$ (qui existe toujours puisque $M$ est contractile), Donaldson remarque que la méthode d'Eells et Sampson s'applique sans plus de travail, et que l'on en déduit l'existence d'une solution de $(*)$ tordue par $\rho$, pour tout temps $t$ appartenant à $[0, \infty[$. De plus $\left|D f_{t}\right|$ est bornée uniformément pour tout temps.

Enfin, si $f_{t}$ converge uniformément vers une application $f_{\infty}$, celle ci est harmonique.

Cette remarque initiale va nous permettre de démontrer aisément nos lemmes. 
3.2. Preuve du Lemme A. Comme le convexe $C$ est contractile, nous pouvons supposer que $f_{0}$ est à valeurs dans $C$. Remarquons que la solution $f_{t}$ de $(*)$ est alors à valeurs dans $C$ pour tout temps.

En effet, la fonction distance $d_{C}$ à $C$ est convexe. Ceci entraîne que, si $g_{t}$ est la fonction $f^{*}\left(d_{C}\right)$, elle satisfait à

$$
\partial g_{t} / \partial t \leq-\Delta g_{t} \text {. }
$$

Dès lors,

$$
\frac{d}{d t} \int_{N} g_{t} d s \leq 0
$$

Enfin puisque $g_{t}$ est positive et $g_{0}$ est nulle, nous en déduisons que $g_{t}$ est toujours nulle.

Raisonnons maintenant par l'absurde, s'il n'existe pas d'application harmonique, notre remarque initiale entraîne que si $x_{0}$ est un point de $\bar{N}$, il existe une suite $\left(t_{n}\right)$ tendant vers l'infini telle que $\left(f_{t_{n}}\left(x_{0}\right)\right)$ tende vers un point $y$ de la sphère à 1 'infini de $M$.

Si maintenant $g=\rho(h)$ est un élément de $\operatorname{Im}(\rho)$, puisque $f_{t}$ est uniformément lipschitzienne, nous avons

uniformément bornée.

$$
d\left(g\left(f_{t_{n}}\left(x_{0}\right)\right), f_{t_{n}}\left(x_{0}\right)\right)=d\left(f_{t_{n}}\left(h\left(x_{0}\right)\right), f_{t_{n}}\left(x_{0}\right)\right)
$$

Ceci entraîne que $g(y)=y$ et la contradiction.

3.3. Preuve du Lemme B. Dans le cas où $M$ est euclidienne, les points à l'infini s'identifient aux directions. Identifions $M$ à $\mathbf{R}^{n}$, et décomposons

$$
M=E \oplus E^{\perp},
$$

où $E$ est l'espace des translations qui commutent à $\operatorname{Im}(\rho)$.

A nouveau, nous partons d'une application $f_{0}$ à valeurs dans $M$ et tordue par $\rho$. Soit $x_{0}$ un point de $\bar{N}, f_{t}$ la solution de $(*)$. Soit enfin, $\left(t_{n}\right)$ une suite de réels tendant vers l'infini et $\left(u_{n}\right)$ une suite de vecteurs de $E$, telles que la suite de points

$$
z_{n}=f_{t_{n}}\left(x_{0}\right)+u_{n}
$$

appartienne à $E^{\perp}$ et converge, éventuellement vers un point à l'infini.

Raisonnons à nouveau par l'absurde. S'il n'existe pas d'application harmonique, notre remarque initiale entraîne, comme précédemment, que $\left(z_{n}\right)$ tend vers un point de la sphère à l'infini de $E^{\perp}$.

Ce point à l'infini determine une direction dont un vecteur directeur est, disons, $v$. Comme précédemment, on montre que le flot géodésique, c'est à dire ici la translation, associé à $v$ commute avec le groupe.

Et nous avons la contradiction car alors $v$ appartient à $E$.

3.4. Remarques. Nous n'avons utilisé nulle part dans cette partie de la démonstration notre hypothèse supplémentaire sur $M$ ce qui nous permet de remarquer que la condition de réductivité est suffisante dans tous les cas (à courbure négative ou nulle) pour assurer l'existence d'une application harmonique. 


\section{BIBLIOGRAPHY}

[BGS] W. Ballman, M. Gromov, and V. Schroeder, Manifolds of non-positive curvature, Progress in Math., vol. 61, Birkhaüser, Boston, 1985.

[C] K. Corlette, Flat G-bundles with canonical metrics, J. Differential Geom. 28 (1988), 361382.

[D] S. K. Donaldson, Twisted harmonic maps and the self-duality equations, Proc. London Math. Soc. 55 (1987), 127-131.

[EO] P. Eberlein and B. O'Neill, Visibility manifolds, Pacific J. Math. 46 (1976), 43-110.

[ES] J. Eells and J. H. Sampson, Harmonic mappings of Riemannian manifolds, Amer. J. Math. 86 (1964), 109-160.

Centre de Mathematiques, Ecole Polytechnique, F-91128 Palaiseau (Cedex), France 